\title{
Galactic Cosmic Rays Modulation in the Vicinity of Corotating Interaction Regions: Observations During the Last Two Solar Minima
}

\author{
Keyvan Ghanbari ${ }^{1,2}$, Vladimir Florinski ${ }^{1,2}$, Xiaocheng Guo ${ }^{3}$ (10), Qiang $\mathrm{Hu}^{1,2}$ (1D), and Richard Leske ${ }^{4}$ \\ ${ }_{1}^{1}$ Department of Space Science, University of Alabama in Huntsville, 301 Sparkman Drive, Huntsville, AL 35899, USA; kg0055@uah.edu \\ ${ }^{2}$ Center for Space Plasma and Aeronomic Research, University of Alabama in Huntsville, 301 Sparkman Drive, Huntsville, AL 35899, USA \\ ${ }^{3}$ State Key Laboratory of Space Weather, National Space Science Center, Chinese Academy of Sciences, Beijing, 100190, People's Republic of China \\ ${ }^{4}$ California Institute of Technology, 1200 E California Boulevard, Pasadena, CA 91125, USA \\ Received 2019 June 16; revised 2019 July 10; accepted 2019 July 10; published 2019 September 2
}

\begin{abstract}
Corotating interaction regions (CIRs) are responsible for short-term recurrent cosmic-ray modulation, prominent near solar minima. Using the OMNI data sets for two periods of low solar activity near the beginning and end of solar cycle 24, superposed epoch analysis was performed on the solar wind plasma features for 53 and 43 events during periods 2007-2008 and 2017-2018, respectively. Turbulent properties of the solar wind were studied using the variance method for each CIR. Power spectra have been constructed for overlapped subintervals in the vicinity of stream interfaces (SIs). Using measured correlation lengths and turbulent energies, parallel and perpendicular diffusion mean free paths for cosmic-ray ions have been inferred based on two distinct theoretical formulations. For the two periods with opposite solar polarities, our results show that unlike solar wind speed, magnetic field strength, flow pressure, and proton density are relatively higher during the latest period. Increased turbulent energy and reduced parallel transport coefficients of energetic particles at the SIs are observed. The diffusion coefficients follow the same trends during both periods. The perpendicular diffusion starts increasing nearly a day before SIs and is higher in the fast wind. Superposed epoch analysis is performed on the $>120 \mathrm{MeV}$ proton count rate obtained from the CRIS instrument on board the $A C E$ spacecraft for the same events. The recorded proton rates have peaks half a day before a SI and reach their minimum more than a day after a SI and have a high anticorrelation with the perpendicular diffusion coefficient.
\end{abstract}

Unified Astronomy Thesaurus concepts: Galactic cosmic rays (567); Solar wind (1534); Corotating streams (314); Interplanetary turbulence (830)

\section{Introduction}

Galactic cosmic rays (GCRs) are highly energetic charged particles originating from sources external to the heliosphere. Inside the heliosphere GCRs are convected with the radially expanding solar wind, experience gradient and curvature drift in a highly inhomogeneous heliospheric magnetic field, scatter off small-scale magnetic irregularities which amounts to diffusive motion, and lose (gain) energy in expanding (compressive) solar wind flows. The resulting transport pattern is a complex superposition of all these processes (Jokipii \& Kota 2001; Langner \& Potgieter 2001). Unlike the diffusive and convective components of the cosmic-ray transport, drift velocity depends on the solar magnetic polarity such that during the negative solar polarity $(A<0)$ positively charged particles enter the inner heliosphere along the heliospheric current sheet (HCS) and exit at high latitudes; the pattern is reversed for the positive solar polarity periods $(A>0)$ (Jokipii \& Thomas 1981; Belov 2000; Gupta et al. 2006; Potgieter \& Vos 2017). As a result of these interactions, the intensity of GCRs with energies $<100 \mathrm{GeV}$ experience variations on multiple timescales that are collectively referred to as solar modulation (Forbush 1937; Burger \& Tanaka 1970; El-Borie 1995; Christian et al. 2000; Pike \& Harrison 2000; Aslam \& Badruddin 2012; Zhao \& Qin 2013). Generally speaking, intensities of GCRs measured in the inner heliosphere are anticorrelated with the level of solar activity. Solar minima are periods of low activity when modulation is weak and GCR intensities are the highest, while during solar maxima modulation is strong and GCR intensities are at their lowest levels.
Gil \& Mursula (2018) compared the two exceptionally strong recurrences of GCR intensities during two solar periods of 2007-2008 and 2014-2015 using neutron monitor data, solar wind data, and coronal extreme ultraviolet images. They found that in the most recent period there was a coronal hole in the southern hemisphere about 3-4 days before each decrease in the CR intensity. By studying the power spectrum of CR intensity, they noticed a broad peak in power spectrum with maximum at a rather long period of about 28.9 days compared to the earlier period with a narrower peak in power spectrum with a maximum at 27.5 days. They also discussed that the difference between the two periods was in the heliolatitude in which the coronal holes causing the enhanced solar rotation recurrence rate were. This also led to different longitudinal GCR distributions. They concluded that the convection by fast high-speed streams was the dominant source of the strong periodicity in cosmic rays.

Aslam \& Badruddin (2012) compared the solar minima and declining phases of solar cycles 20,21, 22, and 23 by investigating the Forbush decreases using the Oulu neutron monitor count rates. They reported that the GCR intensities reached their highest values recorded during the space era, while the sunspot number and IMF strength attained their lowest recorded values at the end of solar cycle 23. Among their findings were the record highest levels of GCR intensities during solar cycle 23 in comparison to the three previous cycles and high correlation between the tilt angle of HCS and cosmicray intensity $(R=-0.92)$. After a statistical investigation of the correlation between the GCR intensity and solar wind parameters, they concluded that despite a larger diffusion 
coefficient and higher drift velocity, the reduced solar wind convection is the most significant factor causing the record high GCR intensity during the solar cycle 23. Leske et al. (2011) also investigated the correlation between the solar wind parameters and the detrended Newark neutron monitor data during 2007 and 2008 and found a high degree of correlation between GCR intensity variations and the solar wind speed.

Corotating interaction regions (CIRs) constitute an important phenomenon causing GCR modulation on relatively short (days to weeks) timescales (Richardson et al. 1996). They are formed by a sequence of slow and fast solar wind streams originating from the low and high latitude regions, respectively. The forgoing slow and the following fast wind in a CIR are separated by a narrow shear layer called the stream interface (SI). CIRs are accompanied by HCS crossings also known as sector boundaries (SBs). On the large heliospheric scales, the HCS is a wavy neutral sheet that separates the opposite magnetic field polarities originating from the northern and southern solar hemispheres. Some argue that the HCS is the main driver of the GCR modulation owing to gradient and curvature drifts along the sheet (Kuzmin et al. 1985; Smith 1990; Thomas et al. 2014), while others have come to the conclusion that the SIs play the dominant role in the modulation process via reduced diffusion caused by enhanced turbulence (Richardson et al. 1996; Intriligator et al. 2001; Richardson 2004; Guo \& Florinski 2016). The latter argue that particle drifts are less important than diffusion effects in the inner heliosphere. The diffusion of the GCRs is caused by small-scale fluctuations in the magnetic field embedded in the solar wind. Intriligator et al. (2001) investigated the particle transport near SIs of two CIRs observed by Pioneer 11 and Ulysses spacecraft. They proposed that in the vicinity of a local current sheet and a velocity shear the magnetic field random walk cannot extend across the discontinuity, which leads to a reduced random walk of the field lines and consequently reduced particle diffusion.

To evaluate the effect of diffusion one needs to study the properties of the solar wind perturbations. The turbulent environment of the solar wind has been studied in detail by multiple groups (e.g., Zank \& Matthaeus 1992; Goldstein 1995; Matthaeus 2000). Power-spectral density is often used to characterize turbulent flows and has been widely employed to study solar wind fluctuations (Matthaeus \& Ghosh 1999; Matthaeus et al. 2007). The power spectrum of magnetic field fluctuations consists of three distinct regions: the energycontaining range at low frequency, the dissipation range at high frequency, and the inertial (turbulent) range in between them. In a broadband view of the turbulence, energy is injected mainly at large scales and transferred toward smaller scales by nonlinear processes, where the kinetic dissipation mechanisms limit the energy transfer (Balogh et al. 1997). The turbulent properties of the solar wind fluctuations and their subsequent radial evolution differ from fast to slow wind during its expansion in the heliosphere. The fluctuations energy, Alfvénicity, the level of Alfvénic correlations and the correlation length are enhanced in the fast wind in comparison with the slow wind. The peculiar characteristics of the solar wind are hard to categorize in a general theoretical framework. Two well-known theories proposed by Kolmogorov and Kraichnan are employed to describe the cascade rate and spectral slopes of the power spectrum for the cases of hydrodynamic and magnetohydrodynamic (MHD) turbulence, respectively (Kolmogorov 1941;
Kraichnan 1965; Tu \& Marsch 1995; Borovsky \& Denton 2010; Bruno \& Carbone 2013). In this study, the main focus is on two properties: the total turbulent energy and the bend-over length. The latter corresponds to the transition point from the energycontaining range and the inertial range in the power spectrum of the solar wind magnetic field fluctuations. The bend-over length therefore is representative of the size of the large-scale eddies in the solar wind plasma (Zank et al. 1996).

Several groups have conducted data-driven or physics-based computer simulations on the heliospheric plasma and consequently the solar modulation of GCR intensities. In these studies MHD equations are solved for the background solar wind plasma parameters, such as the solar wind speed, the large-scale magnetic field $(\boldsymbol{B})$, the proton density, and the proton temperature, using some prescribed boundary conditions depending on the region being simulated (which may span radial distances from a fraction of an astronomical unit (au) to nearly $100 \mathrm{au}$ ). In some earlier work the HCS was assumed to be flat (Ball et al. 2005). However, in recent models the wavy HCS have been implemented to make the model more realistic (Usmanov et al. 2011; Guo \& Florinski 2016). After solving the MHD equations for the solar wind and transport parameters, the Parker transport equation (Parker 1965) is employed to obtain the nearly isotropic probability distribution function of cosmic rays, which can be used to describe the intensity and spectra of the particles under study. This equation reads

$$
\begin{aligned}
& \frac{\partial f}{\partial t}+\left(\boldsymbol{u}_{0}+\boldsymbol{v}_{d}\right) \cdot \nabla f-\nabla \cdot(\kappa \cdot \nabla f) \\
& -\frac{\nabla \cdot \boldsymbol{u}_{0}}{3} \frac{\partial f}{\partial \ln p}=0
\end{aligned}
$$

where $f(\boldsymbol{r}, p)$ is the isotropic part of the cosmic-ray distribution function in phase space as a function of position $r$ and magnitude of momentum $p, \boldsymbol{u}_{0}$ is the mean plasma velocity, $\boldsymbol{v}_{d}$ is the drift velocity of the charged particle due to gradient and curvature drifts, and $\kappa$ is the diffusion tensor that is diagonal (and axisymmetric) in the magnetic field aligned coordinate frame.

For the work presented here, the periods of 2007-2008 and 2017-2018 were studied as the two most recent solar minima. In these two periods, the recurrent solar modulation effects were very prominent. By comparing the solar wind parameters, turbulence properties, and the GCR intensities via superposed epoch (SPE) analysis for these two periods, we investigated how the differences in the solar wind background and turbulence parameters affected the average GCR modulation in the vicinity of CIRs. We also obtained the turbulence and transport parameters around the CIRs to aid future GCR modulation simulations (that will be reported in a later publication). In the next section, the theoretical basis for our computations is presented. Following that, the data and the analysis methods are explained and the results of this study are presented. The paper concludes with a comparison between the two periods and a discussion of the consequences for future GCR modeling.

\section{Theoretical Basis}

Diffusion coefficients describe GCR scattering ion pitch angle and cross-field transport, both of which are determined by the properties of the turbulence in the solar wind. The 
diffusion tensor is defined with the diagonal elements being the parallel and perpendicular components (relative to the mean magnetic field direction). Gradient and curvature drifts may be incorporated as off-diagonal components (Bieber \& Matthaeus 1997; Minnie et al. 2007; Zhao et al. 2018), although this is not common in numerical work, and we do not use this approach (see Equation (1)). For context we use the models of the evolution of turbulence in the solar wind developed initially by the Bartol group (Zhou \& Matthaeus 1990; Zank et al. 1996; Matthaeus et al. 1999). The total turbulent energy $\left(Z^{2}\right)$ is calculated as

$$
Z^{2}=\left\langle\delta u^{2}\right\rangle+\frac{\left\langle\delta B^{2}\right\rangle}{\mu_{0} \rho},
$$

where $\delta u$ is the fluctuating component of the solar wind speed, $\delta B$ is the fluctuating magnetic field, $\mu_{0}$ is the permeability of vacuum, and $\rho$ is the density, which is assumed to be a constant on short scales (the so-called local incompressibility). The bend-over length $\left(l_{b}\right)$ is computed from the power spectrum, which is described in the next section. In order to compute the parallel mean free path (MFP) the standard quasilinear theory (QLT) has been utilized under the assumption that the turbulence is one-dimensional and the wavevectors are parallel to the mean magnetic field (Zank et al. 1998; Giacalone \& Jokipii 1999; le Roux et al. 1999). The parallel diffusion coefficient is computed as

$$
\begin{aligned}
\kappa_{\|} & =\frac{3 v^{3}}{40 \Omega^{2} l_{b} A_{\mathrm{sl}}^{2}} \csc \left(\frac{3 \pi}{5}\right)\left[1+\frac{72}{7}\left(\frac{\Omega l_{b}}{v}\right)^{5 / 3}\right], \\
\lambda_{\|} & =\frac{3 \kappa_{\|}}{v} .
\end{aligned}
$$

In Equation (3), $v$ and $\Omega$ are the speed and the cyclotron frequency of the particle, respectively, and $A_{\mathrm{s} 1}^{2}$ is relative intensity of the "slab" (one-dimensional) component of the turbulence that comprises $20 \%$ of the total, the remainder being made up of the so-called $2 \mathrm{D}$ modes with wavevectors normal to $\boldsymbol{B}$, i.e.,

$$
A_{\mathrm{sl}}^{2}=\frac{\left\langle\delta B_{\mathrm{sl}}^{2}\right\rangle}{B^{2}}, \quad A_{2 \mathrm{D}}^{2}=\frac{\left\langle\delta B_{2 \mathrm{D}}^{2}\right\rangle}{B^{2}} .
$$

The results of the QLT and other models such as the Bieber and Matthaeus model for the perpendicular diffusion coefficient (Bieber \& Matthaeus 1997) are not consistent with numerical particle simulations. However, the nonlinear guiding center theory (NLGC) (Matthaeus et al. 2003; Zank et al. 2004; Shalchi \& Kourakis 2007) were successful in reproducing the numerical simulations and several observations. For relatively high-energy particles such as GCRs the NLGC theory yields the following expression for the perpendicular MFP and diffusion coefficient:

$$
\lambda_{\perp}=\left[\sqrt{3} \pi a^{2} C A_{2 \mathrm{D}}^{2} \lambda_{2 \mathrm{D}}\right]^{2 / 3} \lambda_{\|}^{1 / 3} \quad, \quad \kappa_{\perp}=\frac{\lambda_{\perp} v}{3} .
$$

In Equation (5), it is assumed that the turbulent fluctuations are dominated by the $2 \mathrm{D}$ component leading to the $2 \mathrm{D}$ correlation length, $\lambda_{2 \mathrm{D}}$, being approximately equal to the correlation length $\lambda_{c}$ of the turbulence model (Breech et al. 2008; Chhiber et al. 2017). According to the observations
(Osman \& Horbury 2007; Weygand et al. 2009, 2011) the 2D correlation length is about half of the slab correlation length $\left(\lambda_{s}\right)$, therefore we assume that $\lambda_{s}=2 \lambda_{2 \mathrm{D}}=2 \lambda_{c}$. The correlation length is related to the bend-over length via $l_{b}=1.2618 \lambda_{c}$ for the model magnetic turbulence power spectrum given by Giacalone \& Jokipii (1999). Several methods exist to calculate the bend-over and correlation lengths. As mentioned in the previous section, the bend-over length is the inverse of the wavenumber where the spectrum turns over from the harder energy-containing range to the softer inertial range. The measured frequency spectrum must be converted into the wavenumber spectrum using the Taylor hypothesis (Taylor 1938) by dividing the frequencies by the mean solar wind speed.

\section{Data and Methods}

The 1 minute solar wind data from the OMNIWeb data center (King \& Papitashvili 2002) have been used to obtain the solar wind plasma properties. 53 CIRs from the first period (2007-2008) and 43 from the second period (2017-2018) were identified and studied. The complete list of studied events is given in Table 1. The first period events are the same as those identified by Jian et al. (2011), who used the peak in the total pressure (the sum of thermal and magnetic pressure) to identify the time of the SI passage. The events of the second period were identified by the authors. The CIRs of the second period were identified by eye based on the same signatures as discussed in Jian et al. (2011), including an increase in the solar wind speed, a pile-up of the total pressure, a deflection of the solar wind equatorial angle, an enhancement of proton temperature, and a drop in proton density.

This study is concerned with the trends in transport parameters throughout the entire CIR structure, which requires that the consecutive SIs be separated by more than 5 days, which eliminated $46 \%$ of all events. For the remaining wellseparated CIRs, all solar wind data from 4 days before to 4 days after the SI were analyzed. SPE analysis was performed on solar wind parameters including the solar wind speed, magnetic field magnitude, total and dynamic flow pressure, and proton density. Since most CIRs are accompanied by HCS crossings, it was also required to exclude the SBs from the magnetic field data. For this purpose, SBs have been identified using the total variation denoising (TVD) method (Little \& Jones 2010) on Lambda (defined as the angle between projection of IMF vector on ecliptic and the $X_{\mathrm{GSE}}$ axis) throughout both periods. In this method, noisy data $(y(t))$ composed of a piecewise constant signal $(x(t))$ and a white Gaussian noise $(w(t))$ is treated as an optimization problem to estimate the constant part of the signal $(x(t))$ (Selesnick 2012). The TVD method has been used for a one year period previously by Ford et al. (2016). Using such a long period resulted in some of the closely spaced SBs being optimized out. In this work we applied the same method to shorter intervals (as short as 5 days), which significantly improved the reliability of SB identification. Magnetic field data from $5 \mathrm{hr}$ before to $5 \mathrm{hr}$ after each SB were excluded for the turbulence analysis. Figure 1 illustrates how the method works on the example of four CIR events (62 through 65 in Table 1). The sector boundaries are marked with blue vertical lines.

A conventional approach based on variances was used to analyze the turbulence. The 8 day intervals around each event were subdivided to $6 \mathrm{hr}$ subintervals with $2 \mathrm{hr}(\sim 33 \%)$ overlap, resulting in a total of 94 subintervals. We have chosen $6 \mathrm{hr}$ 
Table 1

CIR Events Studied in This Work

\begin{tabular}{|c|c|c|c|c|c|c|c|c|c|c|c|c|c|c|}
\hline \# & Year & DOY & $\#$ & Year & DOY & \# & Year & DOY & $\#$ & Year & DOY & \# & Year & $\overline{\overline{D O Y}}$ \\
\hline 1 & 2007 & 29.46 & 22 & 2007 & 344.80 & 42 & 2008 & 204.85 & 62 & 2017 & 182.76 & 82 & 2018 & 8.54 \\
\hline 2 & 2007 & 37.75 & 23 & 2007 & 351.22 & 43 & 2008 & 222.22 & 63 & 2017 & 190.16 & 83 & 2018 & 13.88 \\
\hline 3 & 2007 & 43.80 & 24 & 2007 & 361.67 & 44 & 2008 & 231.43 & 64 & 2017 & 202.23 & 84 & 2018 & 88.65 \\
\hline 4 & 2007 & 58.29 & $\ldots$ & $\ldots$ & $\ldots$ & 45 & 2008 & 247.25 & 65 & 2017 & 216.42 & 85 & 2018 & 100.15 \\
\hline 5 & 2007 & 65.46 & 25 & 2008 & 6.26 & 46 & 2008 & 285.30 & 66 & 2017 & 224.14 & 86 & 2018 & 110.30 \\
\hline 6 & 2007 & 71.50 & 26 & 2008 & 14.63 & 47 & 2008 & 293.88 & 67 & 2017 & 229.50 & 87 & 2018 & 120.50 \\
\hline 7 & 2007 & 84.00 & 27 & 2008 & 26.21 & 48 & 2008 & 302.76 & 68 & 2017 & 243.29 & 88 & 2018 & 125.59 \\
\hline 8 & 2007 & 91.00 & 28 & 2008 & 32.72 & 49 & 2008 & 312.50 & 69 & 2017 & 270.30 & 89 & 2018 & 137.30 \\
\hline 9 & 2007 & 98.97 & 29 & 2008 & 42.21 & 50 & 2008 & 320.84 & 70 & 2017 & 278.92 & 90 & 2018 & 143.31 \\
\hline 10 & 2007 & 127.50 & 30 & 2008 & 60.42 & 51 & 2008 & 330.30 & 71 & 2017 & 285.03 & 91 & 2018 & 151.72 \\
\hline 11 & 2007 & 138.50 & 31 & 2008 & 69.38 & 52 & 2008 & 346.13 & 72 & 2017 & 306.30 & 92 & 2018 & 169.26 \\
\hline 12 & 2007 & 153.00 & 32 & 2008 & 86.38 & 53 & 2008 & 357.64 & 73 & 2017 & 311.73 & 93 & 2018 & 186.73 \\
\hline 13 & 2007 & 165.42 & 33 & 2008 & 95.72 & $==$ & $===$ & $===$ & 74 & 2017 & 319.86 & 94 & 2018 & 213.97 \\
\hline 14 & 2007 & 172.38 & 34 & 2008 & 114.22 & 54 & 2017 & 18.21 & 75 & 2017 & 325.02 & 95 & 2018 & 238.59 \\
\hline 15 & 2007 & 192.01 & 35 & 2008 & 124.51 & 55 & 2017 & 54.99 & 76 & 2017 & 331.61 & 96 & 2018 & 286.74 \\
\hline 16 & 2007 & 201.47 & 36 & 2008 & 149.13 & 56 & 2017 & 60.51 & 77 & 2017 & 338.78 & $\ldots$ & $\ldots$ & $\ldots$ \\
\hline 17 & 2007 & 238.86 & 37 & 2008 & 159.38 & 57 & 2017 & 74.55 & 78 & 2017 & 345.47 & $\ldots$ & $\ldots$ & $\ldots$ \\
\hline 18 & 2007 & 257.76 & 38 & 2008 & 166.88 & 58 & 2017 & 80.43 & 79 & 2017 & 351.48 & $\ldots$ & $\ldots$ & $\ldots$ \\
\hline 19 & 2007 & 263.54 & 39 & 2008 & 177.71 & 59 & 2017 & 86.36 & 80 & 2017 & 358.52 & $\ldots$ & $\ldots$ & $\ldots$ \\
\hline 20 & 2007 & 270.67 & 40 & 2008 & 187.76 & 60 & 2017 & 108.96 & $\ldots$ & $\ldots$ & $\ldots$ & $\ldots$ & $\ldots$ & $\ldots$ \\
\hline 21 & 2007 & 276.17 & 41 & 2008 & 194.01 & 61 & 2017 & 121.09 & 81 & 2018 & 1.16 & $\ldots$ & $\ldots$ & $\ldots$ \\
\hline
\end{tabular}

Note. DOY means day of year.

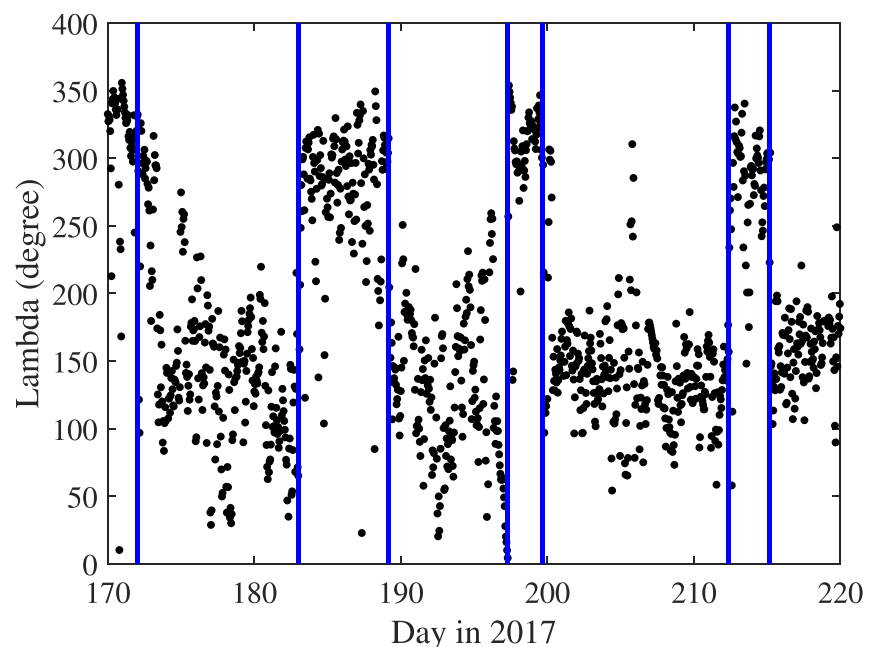

Figure 1. Lambda parameter (see the text) for days 170-220 of 2017. The vertical lines show the SBs identified by the TVD algorithm.

because it is longer than the correlation time of the solar wind turbulence in the spacecraft reference frame, equal to 0.7 to $4 \mathrm{hr}$ (Matthaeus \& Goldstein 1982; Tu \& Marsch 1995; Borovsky $\&$ Denton 2010). This duration allowed us to readily identify the energy-containing range, which was critical for finding the bend-over length. For each $6 \mathrm{hr}$ subinterval, all the solar wind parameters were decomposed into a mean and fluctuating part as

$$
A=\langle A\rangle+\delta A,
$$

where $\langle A\rangle$ is the ensemble average (conventionally replaced with time average using the ergodic assumption) of the corresponding parameter under study in each subinterval, and $\delta A$ is its fluctuation. The magnetic field fluctuations have been used to compute the power spectrum using the Blackman-Tukey method (Blackman \& Tukey 1958). The transition between the energy-containing and inertial ranges is not always distinct. The following procedure was used to infer the bend-over length and the correlation length. After computing the power spectrum for each $6 \mathrm{hr}$ subinterval, the $\log -\log$ spectra in the energycontaining and inertial ranges were approximated with two separate linear fits and the intersection between these two fits was assumed to be at the bend-over frequency, which was converted to bend-over length by multiplying it by the mean solar wind speed in the corresponding subinterval. The variations of the inertial range slope were subsequently studied.

We used the hourly averaged $>120 \mathrm{MeV}$ proton data from the CRIS instrument on board the $A C E$ spacecraft (Stone et al. 1998). We applied fifth- and tenth-order polynomial fits for the first and second periods, respectively, to obtain the mean. After subtracting the fitted curve from the actual data, the detrended data were used for SPE purposes. The hourly averaged data, as well as their corresponding polynomial fits, are shown in Figure 2. SPE analysis was performed on 10 day intervals around SI for each period separately.

\section{Results and Discussion}

The power spectra for subinterval numbers $1,21,32,43,54$, $65,76,87,94$ of event number 1 of Table 1 are shown in Figure 3 . All panels use the power range of $\left(1 \times 10^{-1}-1 \times 10^{4}\right.$ ) $\mathrm{nT}^{2} \mathrm{~Hz}^{-1}$ and the frequency range of $\left(5 \times 10^{-5}-1 \times 10^{-2}\right)$ $\mathrm{Hz}$ for comparison purposes. In these figures, the blue and red lines are the linear fits for the energy-containing and inertial ranges, respectively. The slopes of energy-containing $(\alpha)$ and the inertial range $(\beta)$, the intersection (bend-over) frequency $\left(f_{b}\right)$, and the bend-over length $\left(l_{b}\right)$ are also reported in each panel. The inertial range is approximated in the frequency range of $\left(4.3 \times 10^{-4}-1.9 \times 10^{-3}\right) \mathrm{Hz}$ (Borovsky \& Denton 2010). Considering the fact that the area under the power spectrum is equal to the turbulent energy, one can see from the power spectra that the turbulent energy is lower in the slow wind (subintervals 

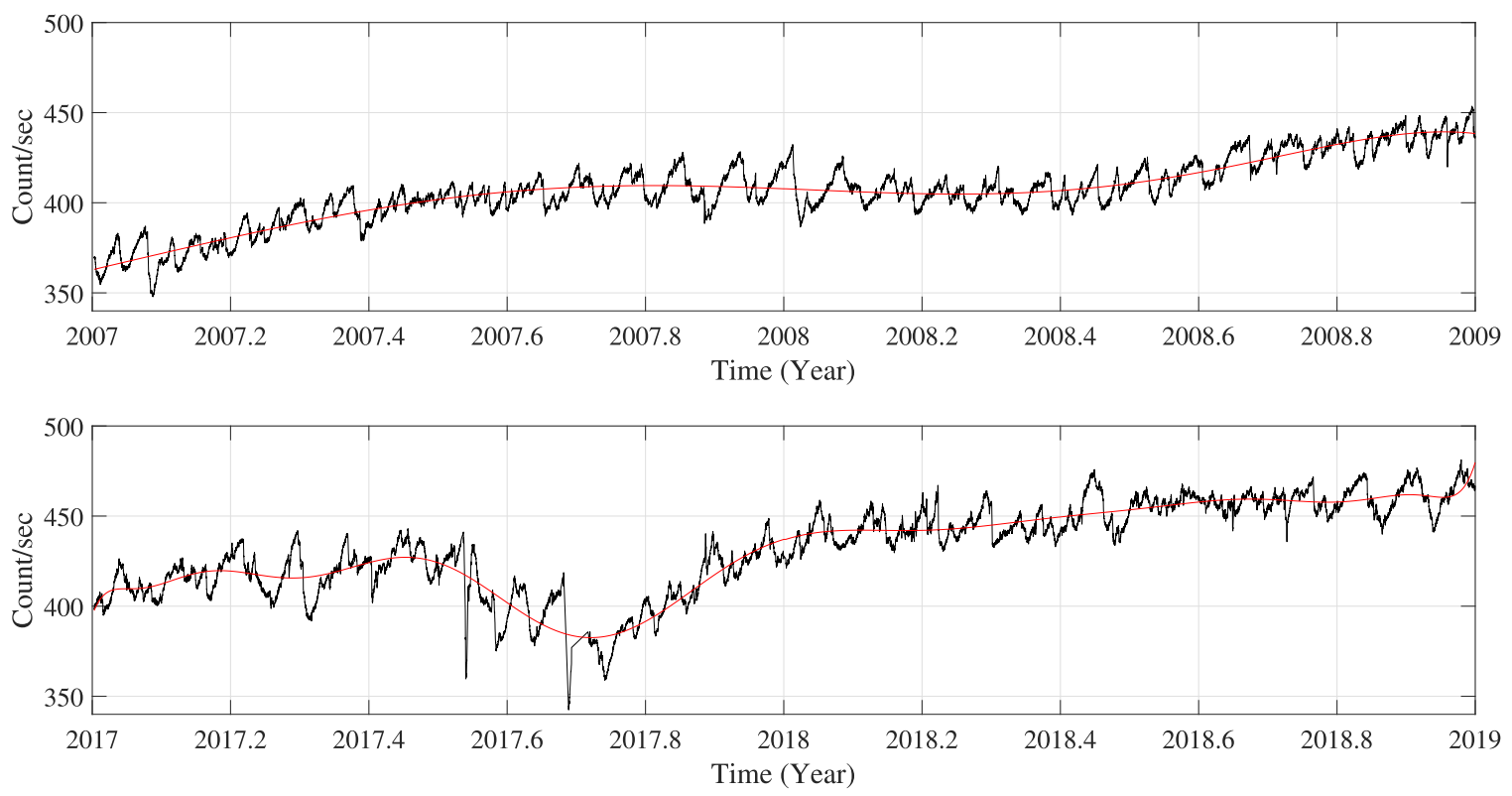

Figure 2. Variation of hourly averaged $>120 \mathrm{MeV}$ proton count $\mathrm{s}^{-1}$ with a fifth-order fit in red throughout the first period (2007-2008; top panel) and a tenth-order fit in red during the second period (2017-2018; bottom panel).

1 and 21). It increases near SI (subinterval 54) and is relatively higher in the fast wind (later subintervals). Note that single events had different behaviors in terms of turbulence characteristics. Also, the intersection (bend-over) frequency is in some cases a little different from the actual bend-over position in the spectrum. One of the advantages of the SPE is that it removes the irrelevant values and keeps the average value and trend of these properties. The slope of the energy-containing range has different behaviors in each spectrum. According to Shalchi et al. (2010), the slope of the energy-containing range has a critical role in determining the perpendicular diffusion coefficient. Three cases for this slope are defined as negative corresponding to a decreasing spectrum, zero corresponding to a constant spectrum, and positive corresponding to an increasing spectrum in the energy-containing range, all of which are evident in our observations in Figure 3.

The SPE analysis of the solar wind speed, IMF magnitude, flow pressure, and proton density are given in Figure 4 . The results for the two periods are given in different colors with the first period in black and the second in blue. The average solar wind speeds in the given time interval around CIRs for both periods have similar trends around the SI. The peak in the speed in both cases starts around a day after the SI and lasts a day and a half in the first period and slightly less than a day for the second period. However, the first period shows a higher speed (by as much as $40 \mathrm{~km} \mathrm{~s}^{-1}$ ) in the fast wind than the second interval. This would cause stronger convection in the first period than the second, which would result in a lower intensity of particles after the SI in the fast wind. As will be discussed below, this situation is observed in the SPE of the proton rates. The average IMF for the whole 8 day interval is stronger for the second period, except a day before the SI. The peak in the IMF magnitude at the SI is as expected. It reaches a value of 10 and $11.5 \mathrm{nT}$ at the peak at the SI, during the first and the second periods, respectively. As the two periods are different in the solar polarities, a comparison is a little complicated. In the first period, particles are drifting in along the HCS through the heliospheric equatorial regions and exit through polar regions. Considering the IMF compression at the
SI as a magnetic barrier, the particles that are swept outward by the CIRs are less likely to be present at the trailing edge of the CIR for the second period due to the stronger IMF barrier. However, the SPE of the proton intensity (see below) is not in agreement with this explanation.

In order to find the SIs, both flow pressure and total pressure are investigated. Both types include the contributions from the different ion species in the solar wind. The total (perpendicular) pressure is the sum of magnetic and thermal pressure (Jian et al. 2011). The average flow pressure is higher for the second period than for the first. The peak at the SI is as high as 6 and 8 $\mathrm{nPa}$ for the first and the second periods, respectively.

The trends in the proton density are similar to those in the IMF. The density is generally higher in the second period compared to the first. For both periods, the slow wind has a proton density of around $5 \mathrm{~cm}^{-3}$ at day 4 before the SI. It increases to approximately 18 and more than $20 \mathrm{~cm}^{-3}$ for the first and second periods, respectively, at the SI. The density drops sharply at the SI and decreases to below $5 \mathrm{~cm}^{-3}$ in the fast wind.

The transport parameters in some cases had rapid fluctuations, necessitating the application of a low-pass filter. The SPE results of the turbulence properties for both periods are presented in Figure 5 using the same color-coding (black for the first period and blue for the second period). As was inferred from the power spectra shown in Figure 3, the turbulent energy for both periods has a peak nearly half a day after the SI at 1500 $\mathrm{km}^{2} \mathrm{~s}^{-2}$. This enhancement in turbulent energy is caused by the shear between the slow and the fast wind at the SI. The slow wind for both periods has turbulent energy less than 500 $\mathrm{km}^{2} \mathrm{~s}^{-2}$. The fast wind in the first period carries more energy until day 4 after the SI in the first period than in the second. These results could be compared with Borovsky \& Denton (2010), who performed an SPE analysis of 27 CIRs between years 2003 and 2008. They have reported the same trend. In their results, the minimum turbulent energy was in the slow wind. In our case, there is a clear minimum for both cases around 1.5 days before the SI. The smooth transition in the turbulent energy from slow to fast wind at the SI has also been 

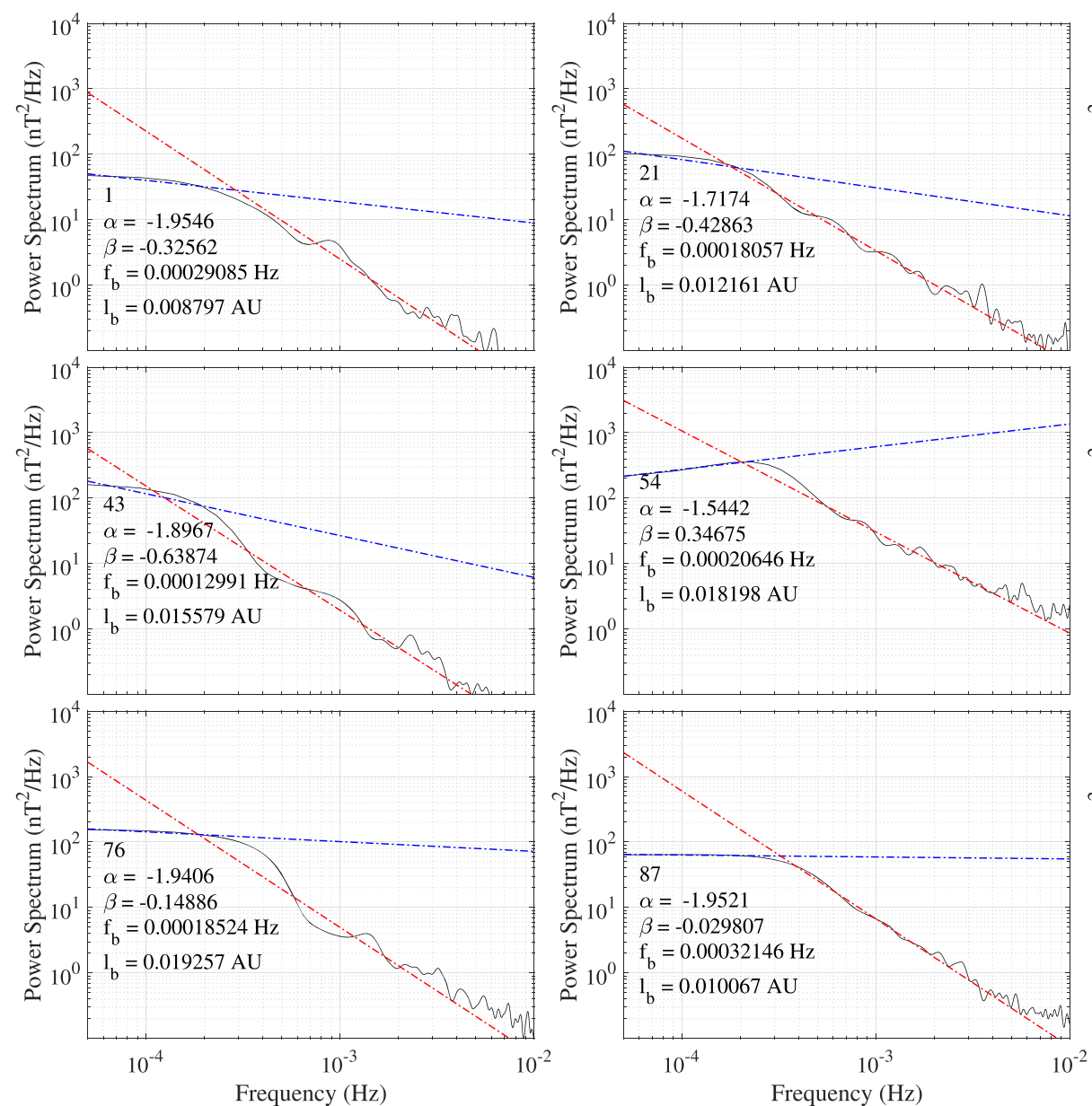
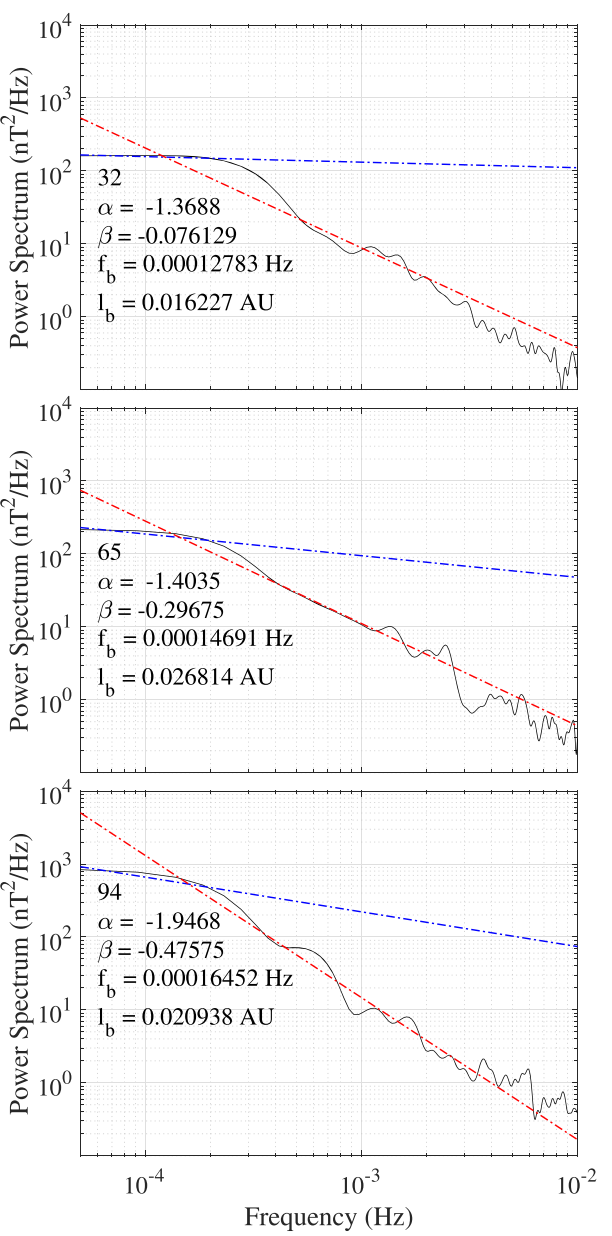

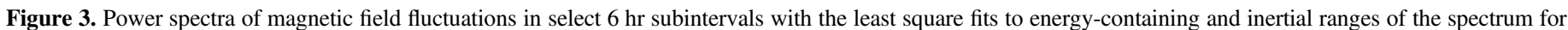

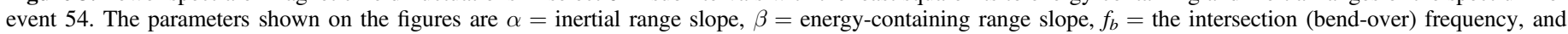
$l_{b}=$ bend-over length.

reported previously (Gosling et al. 1978; Intriligator et al. 2001).

The results of an SPE study of the bend-over length are presented in Figure 5(b). The order of magnitude of the obtained bend-over length is consistent with previous findings. It is again clearly larger in the fast wind than in the slow wind for both periods. The events of the first period have a smaller average bend-over length in the slow wind than the events of the second period. There is a small peak nearly a day after the SI in the second period, while in the first period the bend-over length reaches the maximum 2.5 days after the SI. The method used to obtain the bend-over length here is sensitive to the frequency interval where the linear fits are applied. Even though we are performing a regular least-squares fit for both regions on the log-log plot, one could obtain the weighted fit to give equal weights to points at the low and high ends of the considered inertial range frequency interval. Even so, the obtained bend-over lengths are in good agreement with values reported elsewhere (Matthaeus et al. 2005; Weygand et al. 2009). The two periods are not significantly different from each other.

The parallel and perpendicular diffusion coefficients, as well as their corresponding MFPs computed for $1 \mathrm{GeV}$ protons, are presented in Figures 5(c) and (d). It is worth mentioning that these two parameters are computed using the results of the SPE analysis of the total turbulent energy and bend-over length. The mean parallel diffusion coefficient around the SI is $2.5 \times 10^{22} \mathrm{~cm}^{2} \mathrm{~s}^{-1}$, corresponding to a parallel MFP of 0.06 au. The parallel diffusion coefficient has the minimum exactly at the SI for both periods. The second period has higher parallel diffusion than the first period during the times past the SI. The parallel diffusion coefficient is anticorrelated with the total turbulent energy (correlation coefficient is -0.87 and -0.81 for the first and second periods, respectively), while it has no correlation with the bend-over length $(-0.2$ and -0.03 for the first and second periods, respectively).The reduced diffusion at the SI has also been reported by Intriligator et al. (2001). They stated that the reduced diffusion at the SI is due to reduced perpendicular random walk of the field lines with respect to the shear between the slow and fast wind, which increases the parallel component of the field while keeping the normal components unchanged. This also leads to a change in the field direction within the shear layer. The perpendicular diffusion coefficient and MFP have mean values of $3 \times 10^{20} \mathrm{~cm}^{2} \mathrm{~s}^{-1}$ and $2.3 \times 10^{-3} \mathrm{au}$, respectively. The values start to increase about one day prior to the SI in both periods, however, the first period has a slightly larger perpendicular diffusion coefficient than the second period. The first period reaches its peak at day two after SI, while the second period has a double-peak feature on days one and three after the SI in the fast wind. There is also a local peak in the slow wind for both periods around day three before the SI. This parameter is well-correlated with that of the bend-over length. Zhao et al. (2018) suggested 


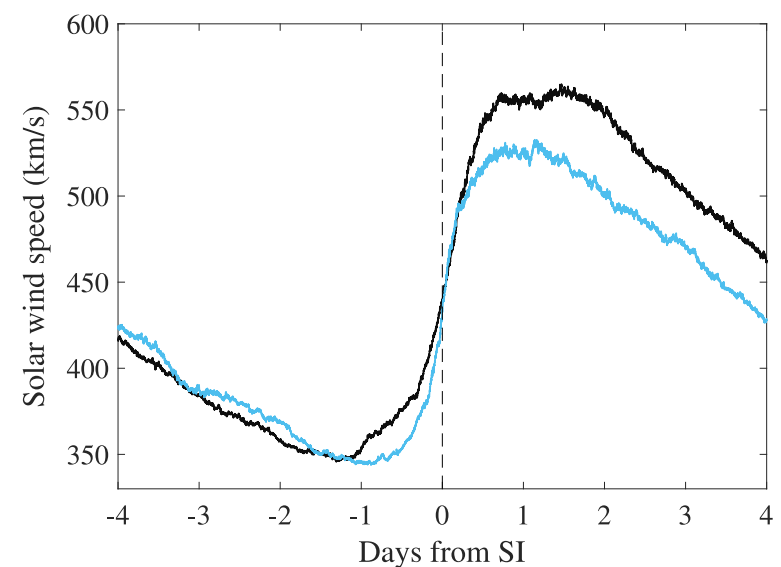

(a)

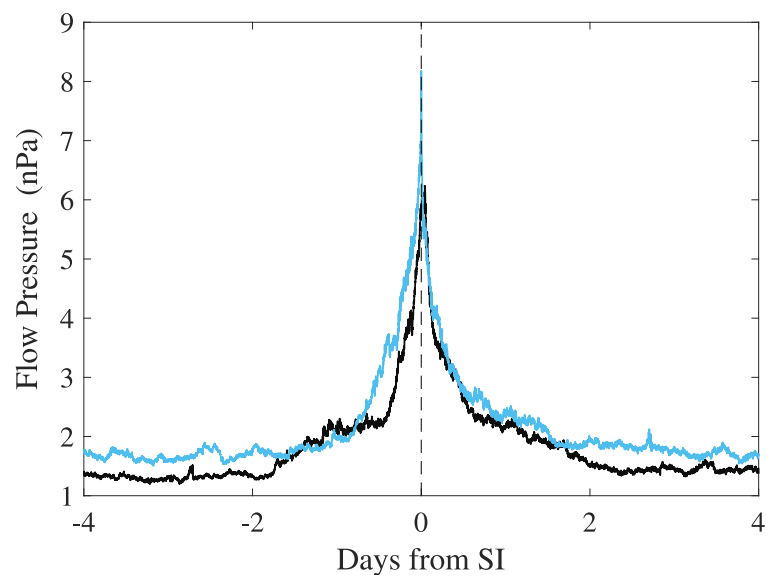

(c)

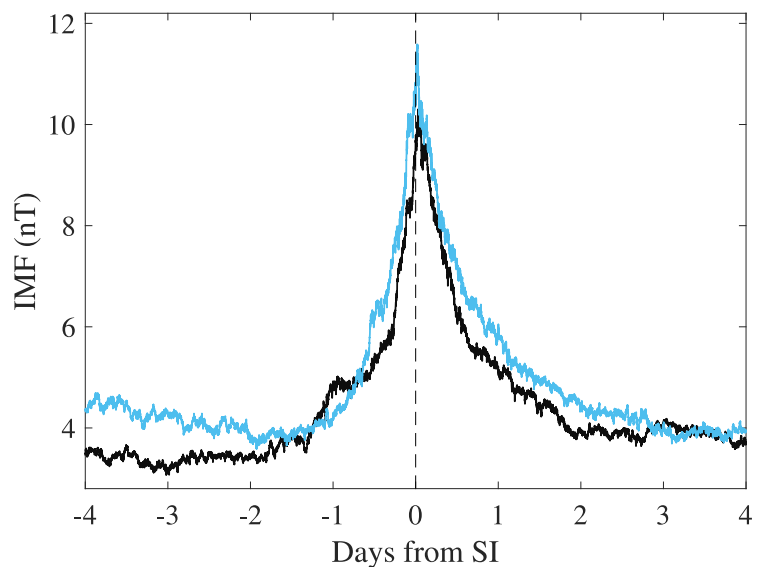

(b)

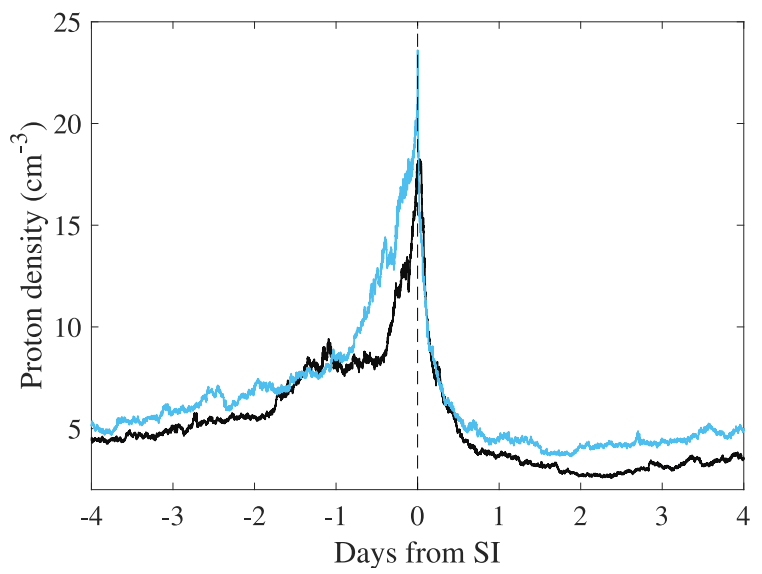

(d)

Figure 4. SPE of (a) solar wind speed, (b) IMF magnitude, (c) solar wind flow pressure, and (d) solar wind proton density, from 4 days before to 4 days after the SI, with the dotted line being the zero-epoch for the first period (black) and second period (blue).

that in addition to the dominance of the parallel MFP, high levels of fluctuations enhance the perpendicular diffusion of CRs and decrease the parallel diffusion if the background IMF strength remains unchanged. They also stated that in case of the changing IMF the described behavior can be obscured. Although the SPE depresses the amplitude of the changes from before and after the SIs, the anticorrelation between the perpendicular diffusion coefficient and the SPE proton count rate $(-0.95$ and -0.85 for first and second periods, respectively) also implies that perpendicular diffusion can be dominant at the SIs and play a major role in modulation.

The ratio of the perpendicular to the parallel diffusion coefficient is shown in Figure 5(e) and has the same trend as the total turbulent energy. Giacalone \& Jokipii (1999) stated that according to their simulations of protons with energies between 1 and $1000 \mathrm{MeV}$ the expected $\kappa_{\perp} / \kappa_{\|} \sim 0.025$, which is the same magnitude as Jokipii \& Kóta (1997). The values obtained here are of the same order of magnitude, with peaks of 0.019 half a day after SI and 0.016 a few hours after the SI for the first and second periods, respectively. The slow wind for both periods has a similar value and trend and the fast wind has a larger ratio during the first period than during the second.

The average spectral slope of the magnetic fluctuations in the inertial range is shown in Figure 5(f). As can be seen, the mean is close to the Kolmogorov approximation of $-5 / 3$. The measured slope is around -1.7 in the slow wind for both periods. The slope starts to increase a day before the SI and reaches the maximum of -1.6 a day after the SI. The trends for both periods are quite similar. Such a trend has also been reported by Borovsky \& Denton (2010). We have also performed an SPE analysis on the energy-containing range slopes which shows that the average slope in the 8 day interval around the SI is -0.2 . As noted earlier, the QLT and NLGC theories were used to compute the transport coefficients. In the case of a parallel diffusion coefficient, the slopes of the energycontaining range and the inertial range are considered to be 0 and $-5 / 3$, respectively. The results show that these assumptions can be challenged by the fact that both slopes vary at each subinterval. These discrepancies should be taken into account.

Considering the average trends of the diffusion coefficients around the SI for both periods, we investigated the proton count rates for the same set of events. The hourly averaged proton count rates for both periods were presented in Figure 2. A notable point about Figure 2 is the higher mean count rate in the second period compared with the first period. In the first period, the hourly averaged count rate starts near 379 counts s$^{-1}$ and increases up to about 450 at the end of the period. At the beginning of the second period, the proton count rate is as high as 400 counts s $^{-1}$ and reaches nearly 470 counts s$^{-1}$ at the end of the period. This can be explained by the fact that during the second period particles penetrating into the heliosphere through the polar region do not "see" as many magnetic barriers as those 


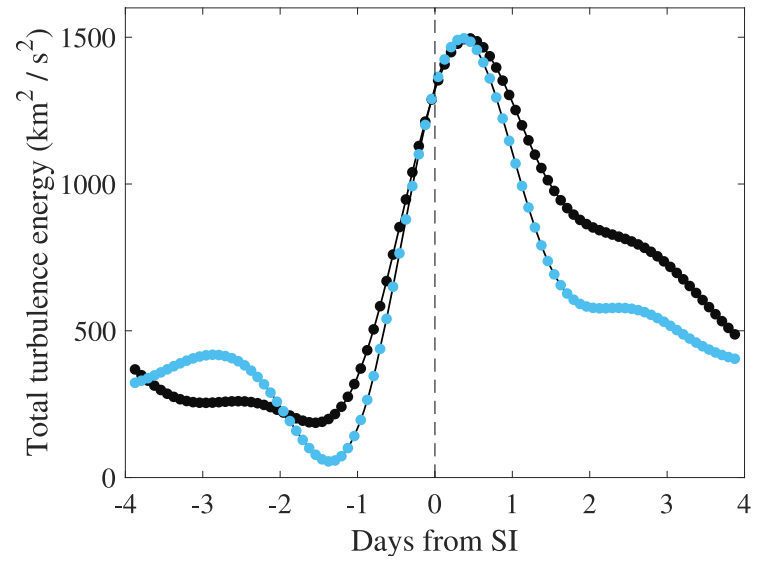

(a)

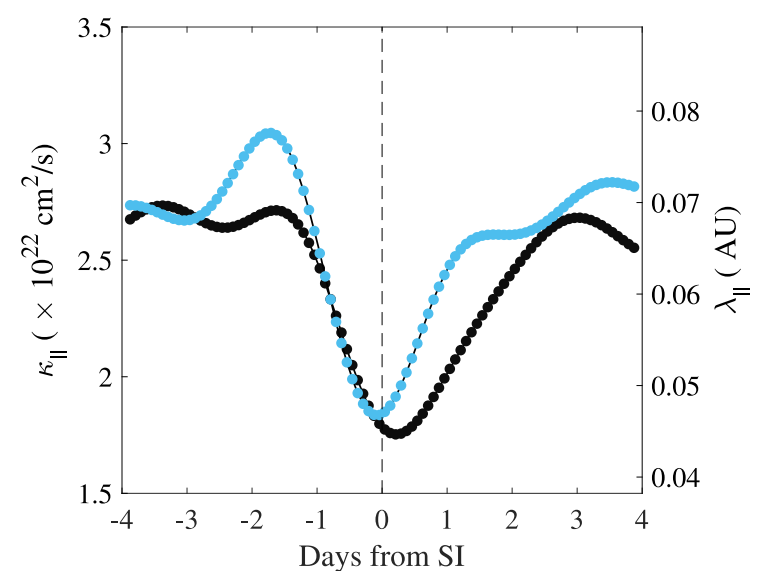

(c)

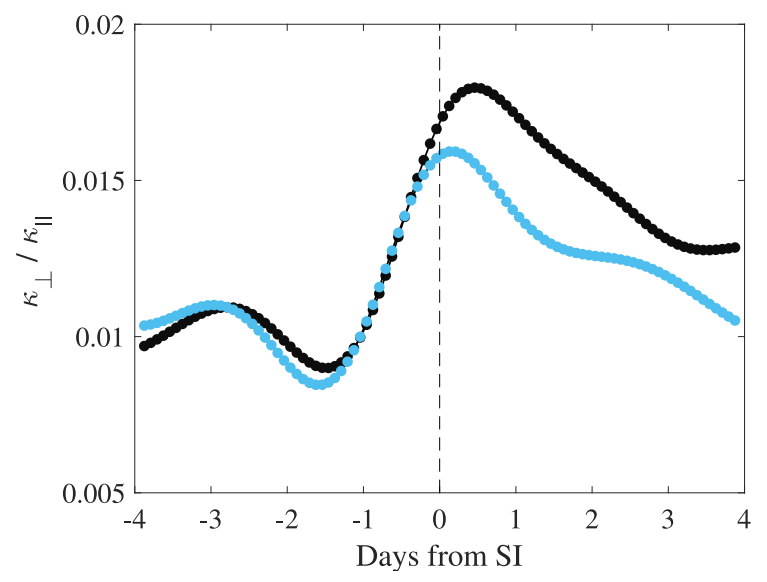

(e)

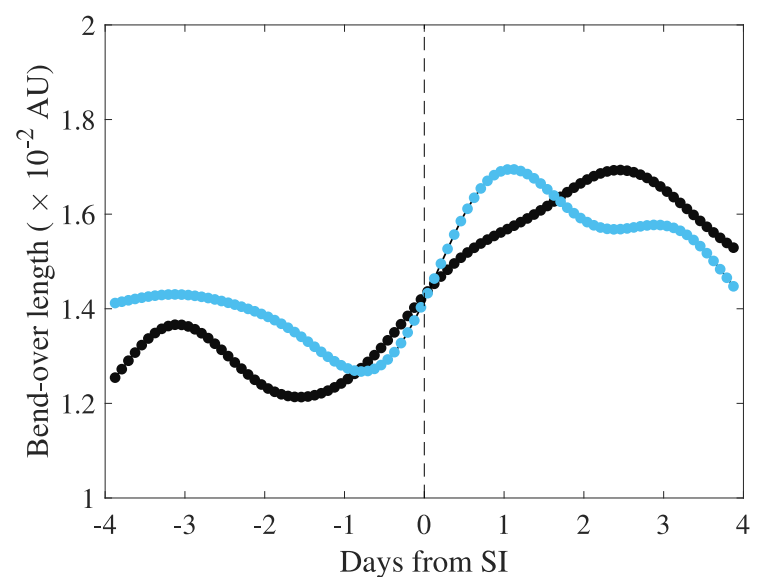

(b)

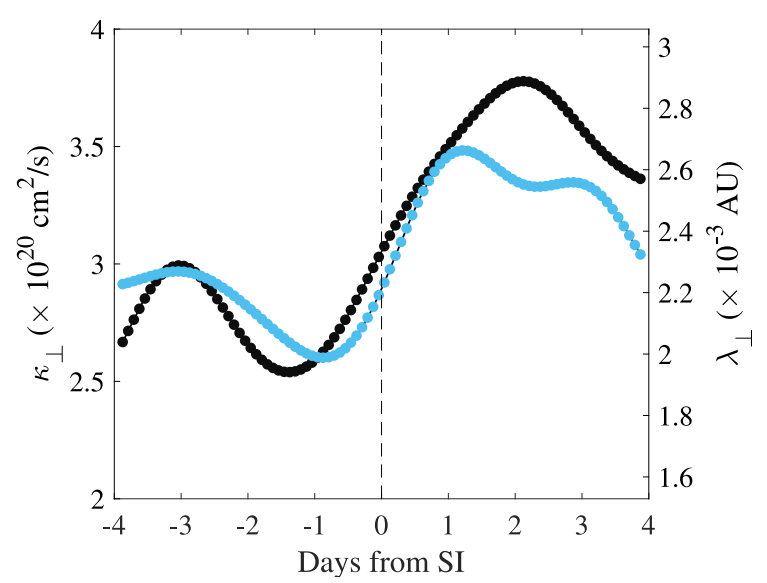

(d)

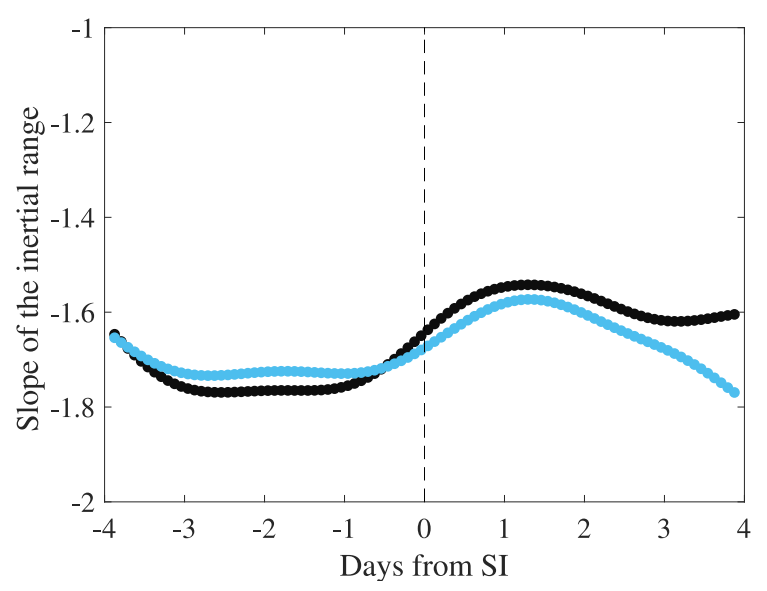

(f)

Figure 5. SPE plots of (a) total turbulent energy, (b) bend-over length (the intersection of linear fits for the energy-containing and inertial ranges, (c) parallel diffusion coefficient (left axis) and parallel MFP (right axis), (d) perpendicular diffusion coefficient (left axis) and the corresponding MFP (right axis), (e) ratio of $\kappa_{\perp} / \kappa_{\|}$, and (f) the slope of the spectrum in the inertial range. Data from 4 days before to 4 days after the SI, shown with the dotted line, were used for this analysis. Results for the first and second periods are shown with black and blue circles, respectively.

during the first period when they travel through the equatorial regions along the HCS and traverse multiple recurrent structures such as CIRs and ICMEs.

Count-rate variations are shown in Figure 6. These were obtained by subtracting the polynomial fits shown with red lines in Figure 2 from the actual data and performing an SPE study for a 10 day interval around the SI. It is worth mentioning that two of the events from the second period (events number 68 and 69) that have been studied for their transport coefficients were excluded from proton-rate SPE analysis because they occurred in the time interval when the GCR data has gaps. The figure has the same color code as Figure 4. It is seen that unlike 


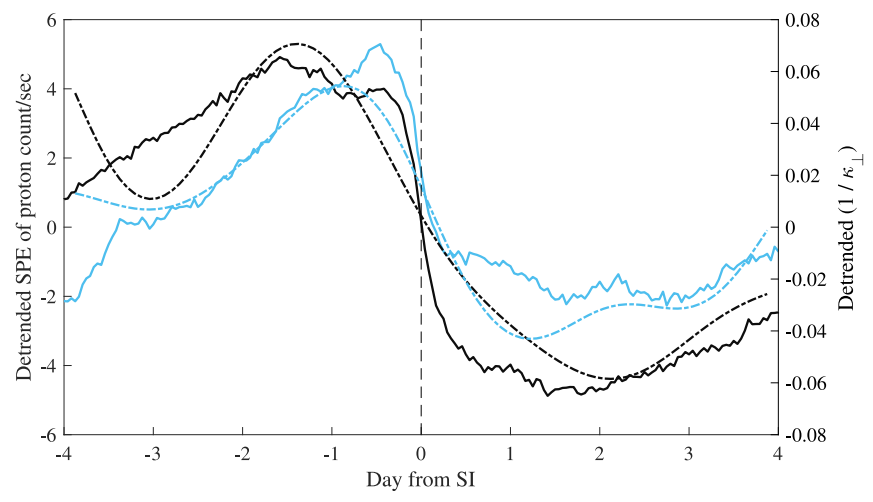

Figure 6. SPE analysis of detrended proton count rates from 4 days before to 4 days after the investigated CIRs for the first period (black solid line), and the second period (blue solid line). The inverse of the perpendicular diffusion coefficient for the same two periods is shown with dashed lines of the corresponding color.

Figure 2 where the amplitude of the changes at each single recurrent event may reach $10 \%$, in the SPE results the amplitude is reduced to some $2 \%$, which is caused by the SPE averaging. The most important feature in both periods is the start of the depression in the proton count rate that begins half a day before the SI. The two periods have different lengths of the peak before the SI, with the first period having a longer peak that starts at day two before the SI and ends half a day before SI, while the second period has a single peak at $1 / 2$ day before the SI. The depression after the SI is also lower in the first period in comparison with the second. The local minimum for the first period occurs at around a day and a half after the SI, while there are two local minima in the proton rates after the SI in the second period.

Figure 6 also plots the inverse of the perpendicular diffusion coefficient for both periods with dashed lines. One can see that the difference in the GCR count rates is strongly correlated with the variations in $\kappa_{\perp}^{-1}$. The long peak before the SI and the deeper minimum after the SI for the first period coincide well with the same features seen in $\kappa_{\perp}^{-1}$. In the second period, the sharper peak closer to the SI and the smoother minimum after the SI are essentially mirrored in the behavior of the inverse diffusion coefficient. While some discrepancy is noticeable at SI-4 days, it is attributable to the determination of the bendover length that becomes less accurate far away from the zeroepoch. Based on the results of this section we argue that out of all solar wind parameters, the perpendicular diffusion plays the dominant role in the modulation of cosmic rays by CIRs.

\section{Summary}

We performed a SPE analysis on both solar wind background and turbulence properties for the two most recent solar minima. The average speed of the fast solar wind has decreased during the most recent period by a factor of $40 \mathrm{~km} \mathrm{~s}^{-1}$. The IMF magnitude, flow pressure, the total pressure, and the proton density are all higher in the most recent period in comparison to the earlier period. The main purpose of this study was to obtain the average values and trends of the turbulence properties around CIRs of two most recent solar minima. In comparing these properties, we found that for both periods the peak in the total turbulent energy occurs half a day after the SI, with fast wind having nearly 2 times greater energy than the slow wind. A major difference between these two periods is the higher levels of turbulence in the fast wind during the first period. Note that the total energy reported here depends on the length of the subintervals used to obtain the power spectra and increases with increasing size of subintervals. The bend-over length is increased in fast wind in comparison to the slow wind in a CIR. The parallel diffusion coefficient is of the order of $10^{22} \mathrm{~cm}^{2} \mathrm{~s}^{-1}$ for both periods, with a dip at the SI. The parallel diffusion coefficient is relatively larger in the second period compared with the first period, especially in the fast wind. The perpendicular diffusion coefficient is, on average, two order of magnitude smaller than parallel. This coefficient shows an increase starting a day before the SI and is higher in the fast wind than in the slow wind. The ratio of the perpendicular to parallel diffusion coefficients has the same features as the turbulent energy, except the peaks do not coincide and the peak in first period is higher and occurs later than that in the second period. The correlation between the perpendicular diffusion coefficient and the SPE of the proton count rate implies that the dominant effect of modulating the GCR around a CIR is the perpendicular diffusion coefficient rather than drift or convection. The obtained trend for both turbulence and transport properties can be used as the reference trends and averages for future simulations of solar modulation of cosmic rays around CIRs.

This research was supported by NASA grant NNX17AB85G. The authors appreciate being granted access to the OMNI data obtained through the GSFC/SPDF OMNIWeb interface at (http://omniweb.gsfc.nasa.gov).

\section{ORCID iDs}

Xiaocheng Guo (i) https://orcid.org/0000-0003-0541-0597

Qiang Hu (iD https://orcid.org/0000-0002-7570-2301

\section{References}

Aslam, O. P. M., \& Badruddin, B. 2012, SoPh, 279, 269

Ball, B., Zhang, M., Rassoul, H., \& Linde, T. 2005, ApJ, 634, 1116 Balogh, A., Dunlop, M. W., Cowley, S. W. H., et al. 1997, SSRv, 79, 65 Belov, A. 2000, SSRv, 93, 79

Bieber, J. W., \& Matthaeus, W. H. 1997, ApJ, 485, 655

Blackman, R. B., \& Tukey, J. W. 1958, BSTJ, 37, 185

Borovsky, J. E., \& Denton, M. H. 2010, JGRA, 115, A10101

Breech, B., Matthaeus, W. H., Minnie, J., et al. 2008, JGRA, 113, A08105

Bruno, R., \& Carbone, V. 2013, LRSP, 10, 2

Burger, J. J., \& Tanaka, Y. 1970, ApJ, 162, 305

Chhiber, R., Subedi, P., Usmanov, A. V., et al. 2017, ApJS, 230, 21

Christian, E. R., Binns, W. R., Cohen, C. M. S., et al. 2000, in AIP Conf. Ser.

528, Acceleration and Transport of Energetic Particles Observed in the

Heliosphere, ed. R. A. Mewaldt et al. (Melville, NY: AIP), 353

El-Borie, M. A. 1995, CaJPh, 73, 642

Forbush, S. E. 1937, PhRv, 51, 1108

Ford, P. E., Florinski, V. A., \& Hu, Q. 2016, AGUFM, SH31B-2590

Giacalone, J., \& Jokipii, J. R. 1999, ApJ, 520, 204

Gil, A., \& Mursula, K. 2018, JGRA, 123, 6148

Goldstein, M. L. 1995, in Proc. Cluster Workshops, Data Analysis Tools and Physical Measurements and Mission-Oriented Theory 371, ESA Special Publication, ed. K.-H. Glassmeier, U. Motschmann, \& R. Schmidt (Paris: ESA), 137

Gosling, J. T., Asbridge, J. R., Bame, S. J., \& Feldman, W. C. 1978, JGR, 83,1401

Guo, X., \& Florinski, V. 2016, ApJ, 826, 65

Gupta, M., Mishra, V., \& Mishra, A. 2006, JApA, 27, 455

Intriligator, D., Jokipii, J., Horbury, T., et al. 2001, JGR, 106, 10625

Jian, L., Russell, C., \& Luhmann, J. 2011, SoPh, 274, 321

Jokipii, J. R., \& Kóta, J. 1997, Proc. ICRC, 8, 151

Jokipii, J. R., \& Kota, J. 2001, Proc. ICRC, 8, 3341

Jokipii, J. R., \& Thomas, B. 1981, ApJ, 243, 1115 
King, J. H., \& Papitashvili, N. E. 2002, AGUSM, SH52A-19

Kolmogorov, A. 1941, DoSSR, 30, 301

Kraichnan, R. H. 1965, PhFl, 8, 1385

Kuzmin, A. I., Samsonov, I. S., \& Samsonova, Z. N. 1985, Proc. ICRC, 5, 250

Langner, U. W., \& Potgieter, M. S. 2001, Proc. ICRC, 9, 3686

le Roux, J. A., Zank, G. P., \& Ptuskin, V. S. 1999, JGR, 104, 24845

Leske, R., Cummings, A., Mewaldt, R., et al. 2011, Proc. ICRC, 11, 194

Little, M. A., \& Jones, N. S. 2010, in Acoustics Speech and Signal Processing (ICASSP), 2010 IEEE Int. Conf., IEEE, 4162

Matthaeus, W. 2000, in Encyclopedia of Astronomy and Astrophysics, ed. P. Murdin (Bristol: IOP), 2306

Matthaeus, W., Bieber, J., Ruffolo, D., Chuychai, P., \& Minnie, J. 2007, ApJ, 667,956

Matthaeus, W. H., Dasso, S., Weygand, J. M., et al. 2005, PhRvL, 95, 231101

Matthaeus, W. H., \& Ghosh, S. 1999, in AIP Conf. Ser. 471, The Solar Wind Nine Conf., ed. S. T. Suess, G. A. Gary, \& S. F. Nerney (Melville, NY: AIP), 519

Matthaeus, W. H., \& Goldstein, M. L. 1982, JGR, 87, 6011

Matthaeus, W. H., Qin, G., Bieber, J. W., \& Zank, G. P. 2003, ApJL, 590, L53

Matthaeus, W. H., Zank, G. P., Smith, C. W., \& Oughton, S. 1999, PhRvL, 82,3444

Minnie, J., Bieber, J. W., Matthaeus, W. H., \& Burger, R. A. 2007, ApJ, 670,1149

Osman, K. T., \& Horbury, T. S. 2007, ApJL, 654, L103

Parker, E. N. 1965, P\&SS, 13, 9

Pike, C. D., \& Harrison, R. A. 2000, A\&A, 362, L21

Potgieter, M. S., \& Vos, E. E. 2017, A\&A, 601, A23
Richardson, I., Wibberenz, G., \& Cane, H. 1996, JGR, 101, 13483

Richardson, I. G. 2004, SSRv, 111, 267

Selesnick, I. 2012, NYU Polytechnic School of Engineering Lecture Notes

Shalchi, A., \& Kourakis, I. 2007, A\&A, 470, 405

Shalchi, A., Li, G., \& Zank, G. P. 2010, Ap\&SS, 325, 99

Smith, E. J. 1990, JGR, 95, 18731

Stone, E. C., Frandsen, A. M., Mewaldt, R. A., et al. 1998, SSRv, 86, 1

Taylor, G. I. 1938, RSPSA, 164, 476

Thomas, S. R., Owens, M. J., Lockwood, M., \& Scott, C. J. 2014, SoPh, 289, 2653

Tu, C.-Y., \& Marsch, E. 1995, SSRv, 73, 1

Usmanov, A. V., Matthaeus, W. H., Breech, B. A., \& Goldstein, M. L. 2011, ApJ, 727, 84

Weygand, J. M., Matthaeus, W., Dasso, S., et al. 2009, JGRA, 114, A07213

Weygand, J. M., Matthaeus, W. H., Dasso, S., \& Kivelson, M. G. 2011, JGRA, 116, A08102

Zank, G., Li, G., Florinski, V., et al. 2004, JGRA, 109, A04107

Zank, G. P., \& Matthaeus, W. H. 1992, JGR, 97, 17189

Zank, G. P., Matthaeus, W. H., Bieber, J. W., \& Moraal, H. 1998, JGR, 103, 2085

Zank, G. P., Matthaeus, W. H., \& Smith, C. W. 1996, in AIP Conf. Ser. 382 , Solar Wind Eight, ed. D. Winterhalter (Melville, NY: AIP), 630

Zhao, L.-L., Adhikari, L., Zank, G. P., Hu, Q., \& Feng, X. S. 2018, ApJ, 856,94

Zhao, L.-L., \& Qin, G. 2013, JGRA, 118, 1837

Zhou, Y., \& Matthaeus, W. H. 1990, JGR, 95, 10291 